\title{
Producción de Diésel Renovable a partir de Aceite de Higuerilla mediante Catalizadores de Níquel-Molibdeno Soportados sobre Alúmina
}

\author{
Lorena Sánchez, Biviana A. Llano y Luis A. Rios \\ Grupo Procesos Químicos Industriales, Facultad de Ingeniería, Universidad de Antioquia UdeA, \\ Calle 70 No. 52-21, Medellín, Colombia (e-mail: lorena.sanchez2@udea.edu.co, \\ biviana.llano@udea.edu.co, luis.rios@udea.edu.co)
}

Recibido Ago. 5, 2016; Aceptado Oct. 4, 2016; Versión final Oct. 28, 2016, Publicado Feb. 2017

\begin{abstract}
Resumen
Se sintetizaron dos catalizadores tipo Ni-Mo soportados sobre $\gamma-\mathrm{Al}_{2} \mathrm{O}_{3}$, con diferentes contenidos másicos de los metales activos, y su actividad catalítica en el proceso de desoxigenación de aceite de higuerilla fue evaluada y comparada con el desempeño obtenido con un catalizador comercial. Previo a las reacciones de desoxigenación, los catalizadores fueron reducidos y sulfurados con $\mathrm{H}_{2} \mathrm{~S}$ producido in-situ. Los catalizadores fueron caracterizados mediante DRX, adsorción-desorción de $\mathrm{N}_{2}$ a $77 \mathrm{~K}$, XRF y TPR. Las pruebas catalíticas fueron llevadas a cabo en un reactor de alta presión (Parr, $500 \mathrm{~mL}$ ), operando en modo semi-batch. El mayor rendimiento másico hacia diésel renovable (C9-C24) fue del $82.9 \%$ y se obtuvo con el catalizador sintetizado que contiene $4.5 \% \mathrm{NiO}$ y $20 \% \mathrm{MoO}_{3}$, evaluado a $350^{\circ} \mathrm{C}$, 90 bares y una concentración del $10 \%$. Además, este catalizador, bajo estas condiciones, presentó una conversión del $100 \%$ y las selectividades más altas hacia C17 (54.4\%) y C18 (20.9\%), así como una menor selectividad a craqueados $(0.83 \%)$.
\end{abstract}

Palabras clave: aceite de higuerilla, desoxigenación, hidroprocesamiento, diésel renovable, $\mathrm{Ni}-\mathrm{Mo} / \gamma$ alúmina

\section{Production of Green Diesel from Castor Oil over Alumina- supported Nickel-Molybdenum Catalysts}

\begin{abstract}
Catalysts of Ni-Mo type with different content of active species (Ni,Mo) were synthesized and supported over $\gamma$-alumina and their catalytic activity in the deoxygenation process of castor oil was evaluated and compared with that obtained with a commercial catalyst. Prior to deoxygenation reactions, the catalysts were reduced and sulfurated with $\mathrm{H}_{2} \mathrm{~S}$ produced in-situ. The catalysts were characterized by XRD, $\mathrm{N}_{2}$ adsorption-desorption at $77 \mathrm{~K}, \mathrm{XRF}$ and TPR. The catalytic tests were carried out in a high-pressure reactor (Parr, $500 \mathrm{~mL}$ ), operating in semi-batch mode. It was found that the highest mass yield to renewable diesel (C9-C24) was $82.9 \%$, obtained with the synthesized catalyst containing $4.5 \% \mathrm{NiO}$ and $20 \% \mathrm{MoO}_{3}$ operating at $350{ }^{\circ} \mathrm{C}, 90$ bars and $10 \%$ catalyst concentration. Besides, this catalysts exhibited $100 \%$ conversion and the highest selectivities to $\mathrm{C} 17$ (54.4\%) and $\mathrm{C} 18$ (20.9\%), as well as the lowest selectivity to cracked products $(0.83 \%)$.
\end{abstract}

Keywords: castor oil, deoxygenation, hydroprocessing, renewable diesel, Ni-Mo/r-alumina 


\section{INTRODUCCIÓN}

El mundo está ingresando en una nueva era energética marcada por preocupaciones de tipo ambiental sumado al agotamiento de las reservas de combustibles fósiles y a la problemática de emisiones contaminantes asociada a su uso, lo cual ha estimulado una amplia búsqueda de alternativas viables para la producción de biocombustibles que permitan remplazar, en alguna medida, el combustible diésel procedente del petróleo (El-Ashry, 2012; Duminda y Sandun, 2013). En este escenario, los biocombustibles derivados de aceites vegetales se presentan como una opción muy atractiva por ser amigables con el ambiente, porque están libres de compuestos de nitrógeno y azufre, presentan propiedades muy similares a las que exhiben los combustibles derivados del petróleo (Liu et al., 2012) y adicionalmente, el método de hidrotratamiento utilizado para su producción puede ser implementado en las refinerías actuales (coprocesamiento) (Priecel et al., 2011a). En Colombia el biodiésel de palma es el biocombustible que se emplea para mezclarlo con el diésel de origen fósil en diferentes porcentajes, no obstante, se han evidenciado algunas restricciones técnicas que dificultan aumentar su porcentaje de mezcla, sumado al problema del empleo del aceite de palma para la producción de biocombustibles dado su competencia directa con la seguridad alimentaria, se hace necesario evaluar otras alternativas de sustitutos del diésel diferentes al biodiésel tradicional. En esta investigación se plantea el uso del aceite de higuerilla (no comestible) para la producción de diésel renovable debido a que no compite con la cadena alimentaria, permite generarle un nuevo valor agregado a una de las biomasas potenciales existentes en el país para la producción de energía (Moncada et al., 2015), y su cultivo puede establecerse en terrenos que han sido marginados o degradados. Cabe resaltar que el diésel renovable no tiene restricción en mezclas con diésel y puede ser empleado en motores sin ninguna modificación previa (Ogunkoya et al., 2015).

Para producir combustibles diésel a partir de materiales orgánicos renovables tales como aceites vegetales, los triglicéridos se pueden hidrogenar en alcanos lineales en una unidad de hidrotratamiento de las refinerías, mediante hidrodesulfuración en presencia de catalizadores convencionales sulfurados (Donnis et al., 2009). El hidrotratamiento ha generado gran interés y ahora es considerado como una de las mejores opciones para los refinadores, productores agrícolas y forestales, debido a que las propiedades de los biocombustibles generados son superiores a las de los productos obtenidos con otros procesos, los costos de inversión para la producción son menores y los impactos sobre el medio ambiente son más bajos (Marker, 2005). Este proceso requiere de hidrógeno para eliminar el oxígeno del aceite en forma de agua, monóxido de carbono o dióxido de carbono. Los productos resultantes son los hidrocarburos respectivos de los ácidos grasos del aceite. El combustible diésel producido a través de este proceso se llama diésel renovable, diésel verde o aceite vegetal hidrogenado, HVO. Una de las ventajas de este proceso radica en que es una tecnología ampliamente desarrollada en la industria del petróleo y del gas (Olusola et al., 2010).

Los catalizadores convencionales empleados en la etapa de desoxigenación corresponden a las formas sulfuradas de Mo o W soportados sobre alúmina, promovidos con Ni o Co. Catalizadores del tipo Ni-Mo y Co-Mo soportados sobre alúmina se han utilizado durante muchas décadas como catalizadores de hidrodesulfuración (HDS) para la eliminación de azufre de las corrientes de petróleo crudo (Priecel et al., 2011a; Kiatkittipong et al. 2013), siendo aptos para ser empleados como catalizadores de hidrotratamiento en la desoxigenación de triglicéridos (Kiatkittipong et al. 2013). Muchos de los efectos como el tipo de soporte, procedimiento de preparación y aditivos (fósforo, potasio o flúor), especies de azufre, y otras variables de reacción, como temperatura, presión y el tipo de catalizador han sido estudiados para la desoxigenación de diversos compuestos oxigenados (Priecel et al., 2011a). A escala de laboratorio se han reportado estudios sobre el hidrotratamiento catalítico de aceite de colza para la producción de alcanos lineales (Donnis et al., 2009); del aceite no comestible de Jatropha utilizando catalizadores soportados en alúmina ( $\mathrm{Pt}-\mathrm{Pd} / \mathrm{Al}_{2} \mathrm{O}_{3}$ y Ni-MoP/Al $\mathrm{O}_{3}$ ) (Gong et al., 2012); usando alúmina (Puralox $\mathrm{SBa} 200$ ) e hidrotalcita (Plural MG70) (Romero et al., 2015); de triglicéridos con catalizadores convencionales sulfurados ( $\mathrm{NiMo} / \mathrm{Al}_{2} \mathrm{O}_{3}$ ) (Huber et al., 2007); de aceite de palma (tipo oleína de palma refinada) para producir diésel bio-hidrogenado (BHD) (Srifa et al., 2014) y del aceite de Girasol (No, 2014). Otros estudios incluyen hidrotratamiento de biomasas como aceites con alto contenido de triglicéridos y los aceites derivados de la licuefacción de alta presión o pirólisis de la biomasa (Gong et al., 2012), aceite de semilla de algodón (Sebos et al., 2009), aceite de colza (Kubička y Kaluža, 2010; Kubička y Horáček, 2011; Priecel et al., 2011b), aceite de soja (Veriansyah et al., 2012; No, 2014) y aceite de girasol (Šimáček et al., 2011). Como regla general, los resultados muestran que se puede lograr una conversión completa de los triglicéridos en condiciones de reacción adecuadas con los catalizadores mencionados.

Es importante resaltar que a la fecha no se evidencian investigaciones acerca del hidrotratamiento del aceite de higuerilla usando catalizadores bimetálicos sulfurados (Ni-MoS/ $/ \mathrm{l}_{2} \mathrm{O}_{3}$ y $\mathrm{Co}-\mathrm{MoS} / \mathrm{Al}_{2} \mathrm{O}_{3}$ ) para la producción de diésel renovable (C9-C24). Solo se reportan tres estudios: el primero está enfocado en la producción de diésel renovable mediante la desoxigenación del biodiésel o metilésteres de ácidos grasos de aceite de higuerilla, utilizando el catalizador $\mathrm{Pd} / \mathrm{C}$, en presencia de hexano a condiciones supercríticas, 
obteniendo como producto principal heptadecano (Meller et al., 2014); el segundo está enfocado en la obtención de combustible tipo Jet empleando Ni/SAPO-11, Ni2P/SAPO-11, Ni/MCM-41, Ni/H-Beta, Ni/ZSM5, Ni/MCM-41 Ni/USY, Ni/USY- MCM-41 y Ni/USY-APTES- MCM-41 como sistemas catalíticos (Liu et al., 2015) y el tercero en la fabricación de diésel renovable y Jet (C8-C15) utilizando $\mathrm{Pt}_{/} \mathrm{Al}_{2} \mathrm{O}_{3} / \mathrm{SAPO}-11$ (Rabaev et al., 2015).

En consonancia con lo anteriormente expuesto, el objetivo principal de esta investigación fue la producción de diésel renovable a partir de la desoxigenación de aceite de higuerilla mediante catalizadores de níquel y molibdeno soportados sobre alúmina, evaluando el efecto de los parámetros de reacción (temperatura y cantidad de catalizador) y del contenido de metales activos Ni-Mo en la pureza, rendimiento, conversión y selectividad hacia diésel renovable (C9-C24), productos de craqueo $(<\mathrm{C} 15)$ y de polimerización $(>\mathrm{C} 20)$. El carácter innovador de la presente investigación reside en buena medida en la utilización de este tipo de catalizadores con la materia prima seleccionada, ya que, como se mencionó previamente, no existen reportes en la literatura sobre hidrotratamiento de aceite de higuerilla con catalizadores tipo Ni-Mo sulfurados.

\section{METODOLOGÍA EXPERIMENTAL}

Se presenta la metodología en tres subsecciones: i) preparación de los catalizadores; ii) caracterización de los catalizadores; y iii) pruebas catalíticas

\section{Preparación de los catalizadores}

Los catalizadores Ni-Mo fueron sintetizados mediante el método de impregnación húmeda utilizando como soporte $\gamma$-alúmina comercial (Merck, $142 \mathrm{~m} 2 / \mathrm{g}$ ). Los precursores metálicos utilizados fueron nitrato de níquel heptahidratado y heptamolibdato de amonio tetrahidratado. En una síntesis típica, se prepararon soluciones acuosas de ambos precursores de forma individual y seguidamente se adicionaron a la $\gamma$-alúmina. La mezcla se dejó bajo agitación a $500 \mathrm{rpm}$ durante 1 hora, posteriormente se evaporó el agua a $110{ }^{\circ} \mathrm{C}$ durante $24 \mathrm{~h}$ en horno y finalmente se calcinó a $500{ }^{\circ} \mathrm{C}$ en aire estático durante $3 \mathrm{~h}$. Los catalizadores sintetizados fueron preparados con la siguiente composición de óxidos de los metales activos: catalizador 1: $6 \% \mathrm{NiO}$ y $25 \% \mathrm{MoO}_{3}$ y catalizador $2: 4,5 \% \mathrm{NiO}$ y $20 \% \mathrm{MoO}_{3}$. El catalizador comercial posee un $3.0 \%$ de $\mathrm{NiO}$ y un $10 \%$ de $\mathrm{MoO}_{3}$, de acuerdo a la ficha técnica del fabricante. Previo a las reacciones de hidrotratamiento, los catalizadores fueron reducidos y sulfurados con $\mathrm{H}_{2} \mathrm{~S}$ producido in-situ bajo las siguientes condiciones: $300{ }^{\circ} \mathrm{C}, 90$ bar de presión de $\mathrm{H}_{2}, 1$ hora y $350 \mathrm{rpm}$ de agitación. Esta operación se llevó a cabo en un Reactor Parr comercial de alta presión (Parr, 500 mL, modelo 4576B), operando en modo batch.

\section{Caracterización de los catalizadores}

Los catalizadores fueron caracterizados mediante el uso de las siguientes técnicas: fluorescencia de rayos $\mathrm{X}(\mathrm{XRF})$, reducción a temperatura programada (TPR), adsorción-desorción con $\mathrm{N}_{2}$ y difracción de rayos $\mathrm{X}$ $(\mathrm{DRX})$.

XRF: La concentración de las especies de $\mathrm{Ni}$, Mo y $\mathrm{S}$ en los catalizadores se determinó mediante fluorescencia de rayos $X$ en un equipo Rigaku Supermini 200, equipado con un tubo de rayos $X$ de $P d$, operando a $50 \mathrm{kVy} 4 \mathrm{~mA}$.

TPR: Se efectuó el análisis TPR para determinar la eficiencia relativa de los catalizadores en la promoción de la reacción y obtener información sobre el tipo de especies reducibles presentes, así como la temperatura a la que ocurre la reducción. Estos ensayos se llevaron a cabo mediante reducción con una mezcla gaseosa de $6 \% \mathrm{H}_{2} / \operatorname{Ar}(50 \mathrm{~mL} / \mathrm{min})$, en un equipo Autochem II modelo 2920.

Adsorción-desorción con $\mathrm{N}_{2}$ a $77 \mathrm{~K}$ : Las áreas superficiales específicas y la porosidad de los catalizadores fueron medidas en un equipo Gemini V, por medio de la adsorción de $\mathrm{N}_{2}$ a $77 \mathrm{~K}$. Previo al análisis las muestras fueron desgasificadas bajo flujo de $\mathrm{N}_{2}$ a $200{ }^{\circ} \mathrm{C}$ durante $10 \mathrm{~h}$.

DRX: Los patrones de difracción de rayos $X$ fueron obtenidos en un equipo Rigaku Miniflex 600 de alta resolución, equipado con un tubo de $\mathrm{Cu}(\lambda=1.5418 \AA)$, operando a $40 \mathrm{kV}$ y $15 \mathrm{~mA}$.

\section{Pruebas Catalíticas}

La actividad catalítica de los dos catalizadores sintetizados y del catalizador comercial (Katalco 61-1T) se evaluó en la reacción de hidroprocesamiento de aceite de higuerilla para la producción de diésel renovable, 
empleando el mismo sistema de reacción de alta presión (Reactor Parr, $500 \mathrm{~mL}$ ) utilizado en el proceso de sulfuración de los catalizadores, operando en modo semi-batch. Esta operación consiste en cargar al reactor todo el aceite y el catalizador, y posteriormente alimentar un flujo continuo de hidrógeno (usando un controlador de flujo másico) para mantener constante la presión mientras se evacuan continuamente los gases de reacción. Para determinar el efecto de los parámetros de reacción en la producción de diésel renovable de aceite de higuerilla, se evaluaron dos temperaturas $\left(300\right.$ y $\left.350^{\circ} \mathrm{C}\right)$ y dos concentraciones de catalizador (5 y $10 \%$ con base en la masa de aceite) y con el fin de analizar la influencia del contenido de metales activos Ni-Mo se estudiaron dos catalizadores sintetizados (catalizador 1: $6 \% \mathrm{NiO}$ y $25 \% \mathrm{MoO}_{3}$ y catalizador 2: $4,5 \% \mathrm{NiO}$ y $20 \% \mathrm{MoO}_{3}$, ambos soportados sobre $\gamma$-alúmina) frente a uno comercial $(3.0 \% \mathrm{NiO}$ y $10 \% \mathrm{MoO}_{3}$ ). La presión de $\mathrm{H}_{2}$ se fijó en 90 bares, el tiempo de reacción fue de 4 horas, la agitación de 350 rpm y la carga de materia prima fue de aproximadamente 100 gramos de aceite de higuerilla para todos los experimentos. Los productos de reacción del hidrotratamiento del aceite de higuerilla se caracterizaron mediante Cromatografía Gaseosa (CG) con un detector de lonización de Llama (FID), en un equipo Agilent 7890A con una columna capilar Agilent DB5-HT y metil estearato como estándar interno. Además, se determinaron los siguientes parámetros: índice de acidez (IA) (NTC 218, 2011), índice de saponificación (IS) (NTC 335, 1998), contenido de hidrocarburos y pureza. También se efectuó el cálculo del rendimiento másico hacia diésel renovable (YDR), el rendimiento hacia productos craqueados y polímeros, la selectividad a diésel renovable C9-C24, a heptadecano y octadecano y la conversión. A continuación se describe las fórmulas matemáticas utilizadas en el cálculo de los parámetros mencionados; en la sección notación se encuentra la definición de todos los términos involucrados en las ecuaciones.

$Y D R=Y P L{ }^{*}$ Pureza $D R=\frac{g \text { DR }}{100 g A A}$

$Y P L=\frac{g P L}{g A A}$

Pureza $D R=\frac{g \text { DR }}{100 \mathrm{~g} \mathrm{PL}}$

$Y C=Y P L * P u r e z a C=\frac{g ~ C}{100 g \text { AA }}$

Pureza $C=\frac{g \mathrm{C}}{100 \mathrm{~g} \mathrm{PL}}$

$Y P=Y P L * P u r e z a P=\frac{g P}{100 g ~ A A}$

Pureza $P=\frac{g \mathrm{P}}{100 \mathrm{~g} \mathrm{PL}}$

$C=100-\left[\frac{[(I S P L-I A P L) * g P L]}{\left[(\text { IS AA }- \text { IA AA })^{*} g A A\right]} * 100\right]$

$\mathrm{SDR}=\frac{\mathrm{YDR}}{\mathrm{C}} * 100$ 


\section{RESULTADOS Y DISCUSIÓN}

Se presentan los resultados y se discuten sus alcances en dos subsecciones: caracterización de los catalizadores y pruebas catalíticas

\section{Caracterización de los catalizadores}

En la figura 1 se observa los difractogramas de los catalizadores sintetizados y del catalizador comercial, después de ser sometidos al proceso de reducción y sulfuración.

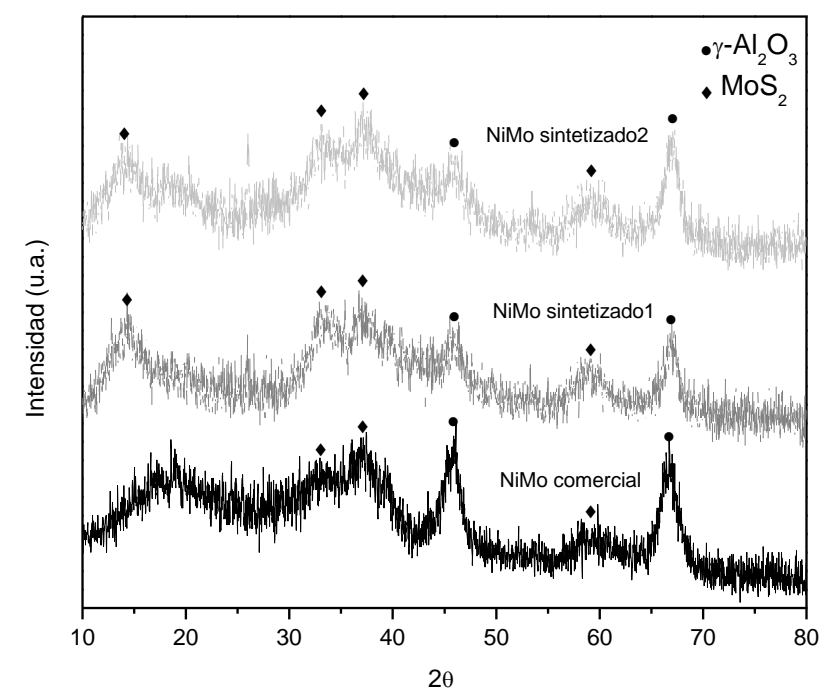

Fig. 1: Difractograma de rayos $\mathrm{X}$ de los catalizadores $\mathrm{NiMoS} / / \gamma-\mathrm{Al}_{2} \mathrm{O}_{3}$ sintetizados y del comercial.

Para los tres catalizadores se observa, de forma clara, los picos principales de difracción de la forma cúbica de la $\gamma$-alúmina, a $2 \theta 46^{\circ}$ y $67^{\circ}$ (JCPDS 10-425). No se observa la presencia de la fase cristalina ortorrómbica de $\mathrm{MoO}_{3}$ cuya principal reflexión aparece a $2 \theta 27.3^{\circ}$, lo cual indica que el Mo se encuentra altamente disperso y además está presente principalmente en la forma sulfurada $\mathrm{MoS}_{2}$ (Priecel et al., 2011a). En concordancia con esta última afirmación, se observan las reflexiones correspondientes a MoS 2 hexagonal (JCPDS $37-1492)$ en $2 \theta 14.4^{\circ}(002), 33.5^{\circ}(100), 39.5^{\circ}(103)$ y $58.6^{\circ}(110)$ (Lai et al., 2013; Wang et al., 2014). No es clara la presencia de la fase $\mathrm{Ni}_{2} \mathrm{~S}_{3}\left(2 \theta=22^{\circ}, 31^{\circ}, 38^{\circ}, 50^{\circ}\right.$ y $\left.56^{\circ}\right)$ (Wang et al., 2014) ni de la forma oxidada $\mathrm{NiO}\left(2 \theta=43.5^{\circ}\right.$ y $\left.63^{\circ}\right)$ (Priecel et al., 2011a), indicando que estas estructuras pueden estar altamente dispersas, amorfas o en forma microcristalina, especialmente el sulfuro de níquel. Los picos débiles y amplios indican una alta dispersión después del proceso de sulfuración, así como la no formación de agregados de partículas después de la sulfuración (Lai et al., 2013).

Las propiedades de superficie medidas por el método adsorción-desorción de $\mathrm{N}_{2}$ a $77 \mathrm{~K}$ a los catalizadores sin sulfurar, indican que el catalizador comercial posee un área superficial específica muy superior a la de los materiales sintetizados, no obstante estos tienen un área suficiente para efectuar la reacción de hidrotratamiento, como se puede comprobar más adelante. En efecto, los resultados catalíticos obtenidos con los catalizadores sintetizados sugieren que se podría lograr un desempeño mucho mejor si se usará un soporte con mayor área superficial. Este aspecto cobraría mayor relevancia en un proceso implementado a escala industrial. La disminución del área superficial en los catalizadores sintetizados con respecto a la del soporte $\left(142 \mathrm{~m}^{2} / \mathrm{g}\right)$ obedece a un bloqueo parcial de los mesoporos con la incorporación de los metales en la estructura, debido a que estos contribuyen más al área total. De forma lógica este parámetro decae más en el material con mayor contenido de $\mathrm{Ni}$ y Mo (catalizador sintetizado 1). Con respecto al volumen y el diámetro de poro los valores obtenidos se corresponden con una estructura mesoporosa, mientras que el diámetro de poro es lo suficientemente grande $(\sim 5 \mathrm{~nm})$ para permitir la difusión de las moléculas de triglicéridos. En un estudio de producción de biodiésel a partir de aceite de girasol, Lukić et al. (2009) reportaron que el diámetro crítico de la molécula de triglicérido, definido como el cilindro más pequeño a través del cual ésta pasa sin distorsión, es de aproximadamente $2 \mathrm{~nm}$. Es de esperarse que en sistemas catalíticos, en los cuales el tamaño de la molécula reaccionante y de los poros del catalizador sea comparable, se presenten restricciones al proceso de difusión y que en buena medida un contacto eficiente entre el reactivo y los sitios activos suceda en la superficie externa del catalizador. 
Tabla 1: Propiedades fisicoquímicas de los catalizadores sintetizados y del comercial. *Determinado por el método BJH aplicado al tramo de desorción de la isoterma.

\begin{tabular}{lccc}
\hline \multicolumn{1}{c}{ Catalizador } & Ni-Mo comercial & Ni-Mo sintetizado 1 & Ni-Mo sintetizado 2 \\
\hline Área BET, $\mathrm{m}^{2} \mathrm{~g}^{-1}$ & 248 & 70 & 80 \\
\hline $\mathrm{Vp}^{*}, \mathrm{~cm}^{3} \mathrm{~g}^{-1}$ & 0,67 & 0,13 & 0,15 \\
\hline $\mathrm{Dp}, \mathrm{nm}$ & 8,3 & 5,3 & 5,3 \\
\hline $\mathrm{NiO} \%$ & 5,5 & 6,5 & 4,5 \\
\hline $\mathrm{MoO}_{3}, \%$ & 13,9 & 32,2 & 21,1 \\
\hline $\mathrm{SO}_{3}, \%$ & 9,6 & 10,0 & 10,9 \\
\hline $\mathrm{Al}_{2} \mathrm{O}_{3}, \%$ & 69,9 & 50,9 & 63,4 \\
\hline
\end{tabular}

Los análisis de TPR se efectuaron a los catalizadores sintetizados y al catalizador comercial. La forma de los perfiles de reducción fue la misma para los tres catalizadores, evidenciándose tres picos de reducción ubicados en $457^{\circ} \mathrm{C}, 489^{\circ} \mathrm{C}$ y $790^{\circ} \mathrm{C}$, no obstante la intensidad de los picos fue diferente y se incrementó en función del contenido de Mo en los catalizadores. Los cambios observados en los perfiles son debido a los procesos de reducción de $\mathrm{Mo}^{6+} \rightarrow \mathrm{Mo}^{4+}$ y Mo ${ }^{4+} \rightarrow \mathrm{Mo}^{0}$. El primer pico ubicado en $457^{\circ} \mathrm{C}$ puede ser adscrito a la reducción de Mo en especies de Mo poliméricas octahedrales. La fase $\mathrm{Mo}^{5+}$ se forma y se reduce simultáneamente hacia $\mathrm{Mo}^{4+}$. El pico que se observa a $660^{\circ} \mathrm{C}$ es resultado de los complejos procesos de reducción de estos compuestos. A $790^{\circ} \mathrm{C}$ se observa otro importante pico de reducción que se relaciona con la difícil reducción de especies de Mo tetrahedrales, y corresponden al proceso $\mathrm{Mo}^{4+} \rightarrow \mathrm{Mo}^{0}$.

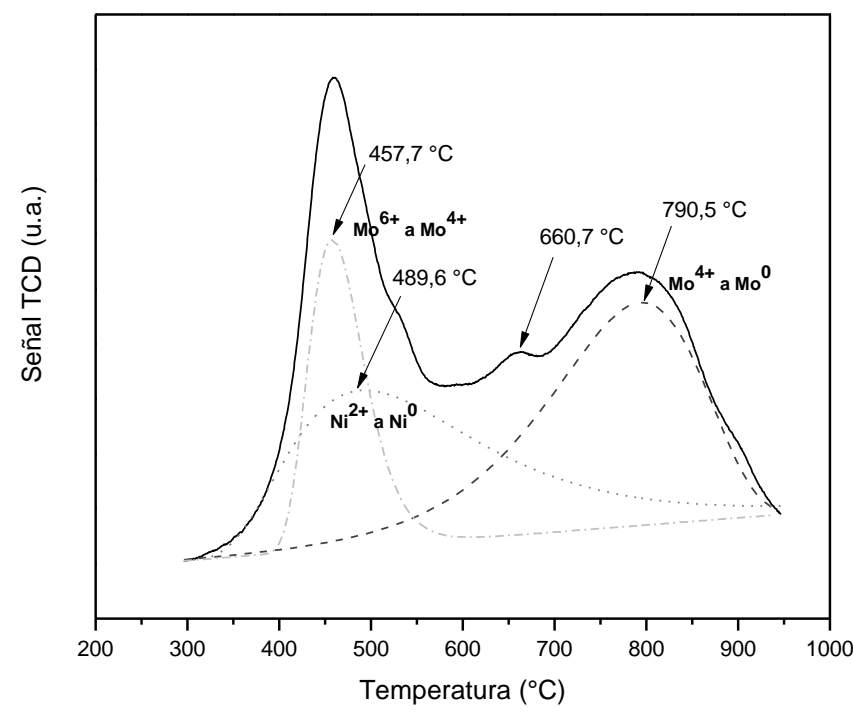

Fig. 2: TPR del catalizador sintetizado 1 sin sulfurar, $\mathrm{Ni}-\mathrm{Mo} / \gamma-\mathrm{Al}_{2} \mathrm{O}_{3}\left(6 \% \mathrm{NiO}, 25 \% \mathrm{MoO}_{3}\right)$.

Al efectuar un proceso de deconvolución al perfil de reducción de los catalizadores se observa un pico adicional en $489^{\circ} \mathrm{C}$, el cual se corresponde con la reducción de las especies de $\mathrm{Ni}^{2+}$. La adición de níquel a la alúmina promueve la reducción de las especies de Mo, ocasionando que este proceso transcurra a menores temperaturas (Domínguez-Crespo et al., 2007). La figura 2 muestra el análisis TPR del catalizador sintetizado 1.

\section{Pruebas catalíticas}

En las tablas 2 y 3 se reportan los resultados de la caracterización de los productos obtenidos a partir del aceite de higuerilla, bajo las diferentes condiciones experimentales evaluadas, así como los resultados de rendimiento, conversión, selectividad y pureza. La caracterización de productos se hizo por análisis cromatográfico y mediante la medición de los índices de saponificación (IS) y de acidez (IA), los cuales permiten determinar la presencia de materia prima sin reaccionar (ésteres) y de intermedios de reacción (ácidos grasos). 


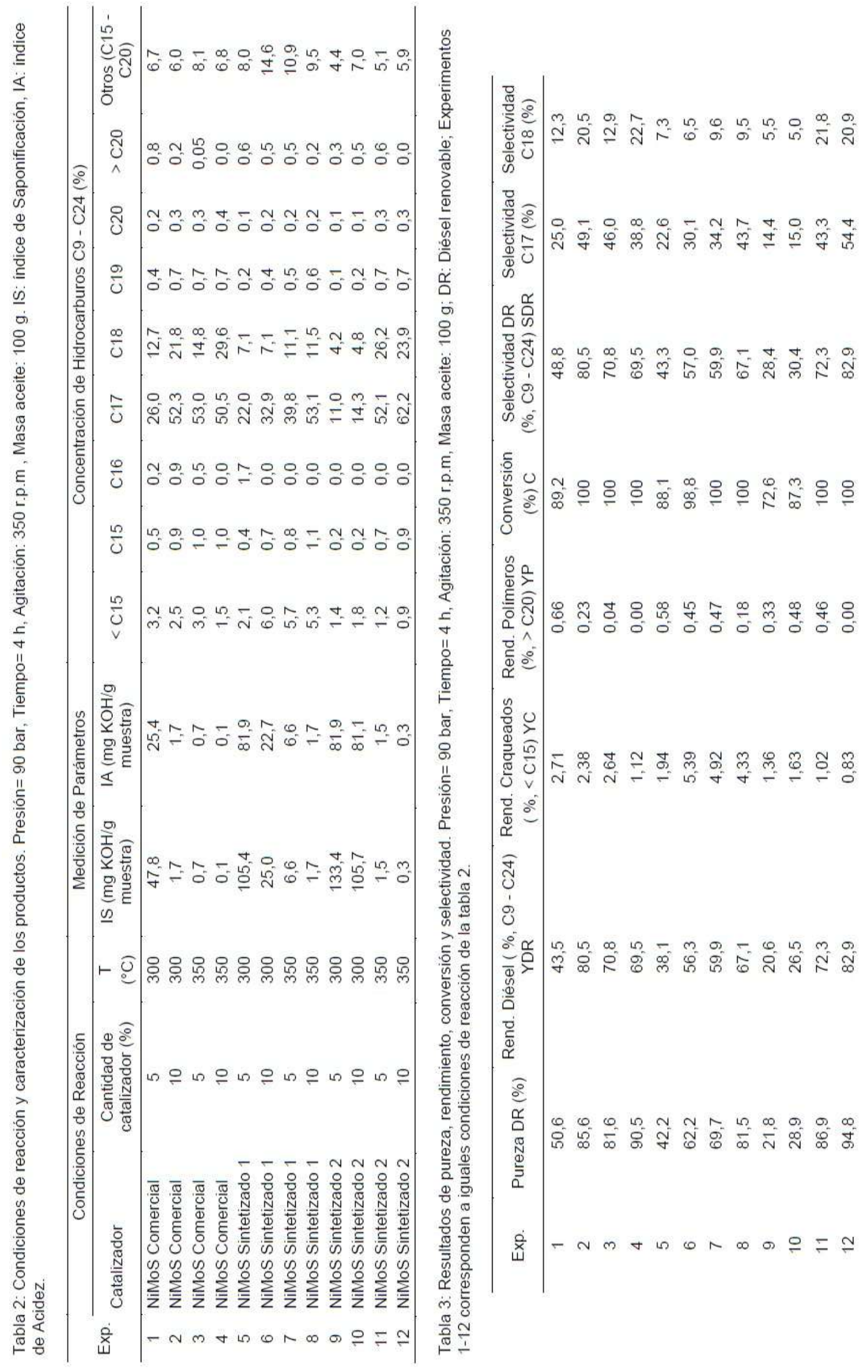


En las tablas 2 y 3 se puede observar que, para las condiciones evaluadas, la temperatura de reacción es el factor de más peso en la desoxigenación del aceite de higuerilla. Para todos los catalizadores usados el índice de saponificación y de acidez disminuyó con el aumento de la concentración de catalizador, siendo menores los valores obtenidos con el aumento de la temperatura. Respecto al contenido de hidrocarburos para todas las condiciones de reacción evaluadas el porcentaje de heptadecano fue mayor al de octadecano al igual que la selectividad hacia estos compuestos, lo que indica que las principales rutas de reacción por la que se formaron los productos fueron descarboxilación $\left(\mathrm{DCO}_{2}\right)$, descarbonilación (DCO) y en menor porcentaje hidrodesoxigenación (HDO) (Srifa et al., 2014). La pureza y el rendimiento másico hacia diésel renovable (C9-C24) en la mayoría de los casos aumentan con el incremento de la concentración del catalizador en la reacción y la temperatura del proceso, excepto para el catalizador comercial donde a 350 ${ }^{\circ} \mathrm{C}$ y $10 \%$ de catalizador se presentó un menor rendimiento $(69,5 \%)$ en comparación con el punto realizado a igual temperatura usando $5 \%$ de catalizador (70,8\%) como se puede apreciar en la figura 3 . En relación a los tres sistemas catalíticos empleados se puede decir que el mayor rendimiento hacia productos craqueados se presentó en los productos obtenidos a partir del catalizador sintetizado 1 y para todos las condiciones estudiadas el rendimiento hacia polímeros fue relativamente bajo y menor al $1 \%$.

El producto con mayor selectividad y rendimiento másico hacia diésel renovable $(82,9 \%)$, mejor pureza $(94,8 \%)$, menor rendimiento hacia productos craqueados $(0,83 \%)$ y polímeros $(0,0 \%)$, con una conversión del $100 \%$, selectividad hacia heptadecano $(54,4 \%)$ y hacia octadecano $(20,9 \%)$ fue el obtenido con el catalizador sintetizado 2 , operando a $350^{\circ} \mathrm{C}, 90$ bar y una concentración de catalizador del $10 \%$. La figura 3 evidencia un aumento gradual del rendimiento hacia diésel renovable en función de la temperatura de reacción del proceso y de la concentración del catalizador utilizado, con excepción del sistema catalítico comercial donde el aumento de temperatura no favoreció el rendimiento a diésel renovable, lo cual permite inferir que la composición de este catalizador genera una mayor cantidad de productos gaseosos (propano, $\mathrm{CO}_{2}$, CO, metano y vapor de agua). Efectivamente, de forma experimental se observó que para este catalizador el rendimiento volumétrico de los productos líquidos, a $350{ }^{\circ} \mathrm{C}$ y $10 \%$ de concentración de catalizador fue el menor de todos los sistemas evaluados, lo cual incidió de forma negativa sobre el rendimiento hacia el biocombustible.

Con el fin de observar cómo influye el tipo de catalizador en el proceso de desoxigenación del aceite de higuerilla a condiciones de $350{ }^{\circ} \mathrm{C}, 90$ bar, 4 horas y $350 \mathrm{rpm}$, específicamente la concentración de las especies sulfuradas de molibdeno $\left(\mathrm{MoS}_{2}\right)$, en la figura 4 se muestra como varía el rendimiento másico hacia diésel renovable en función del contenido de $\mathrm{MoO}_{3}$ en los catalizadores.

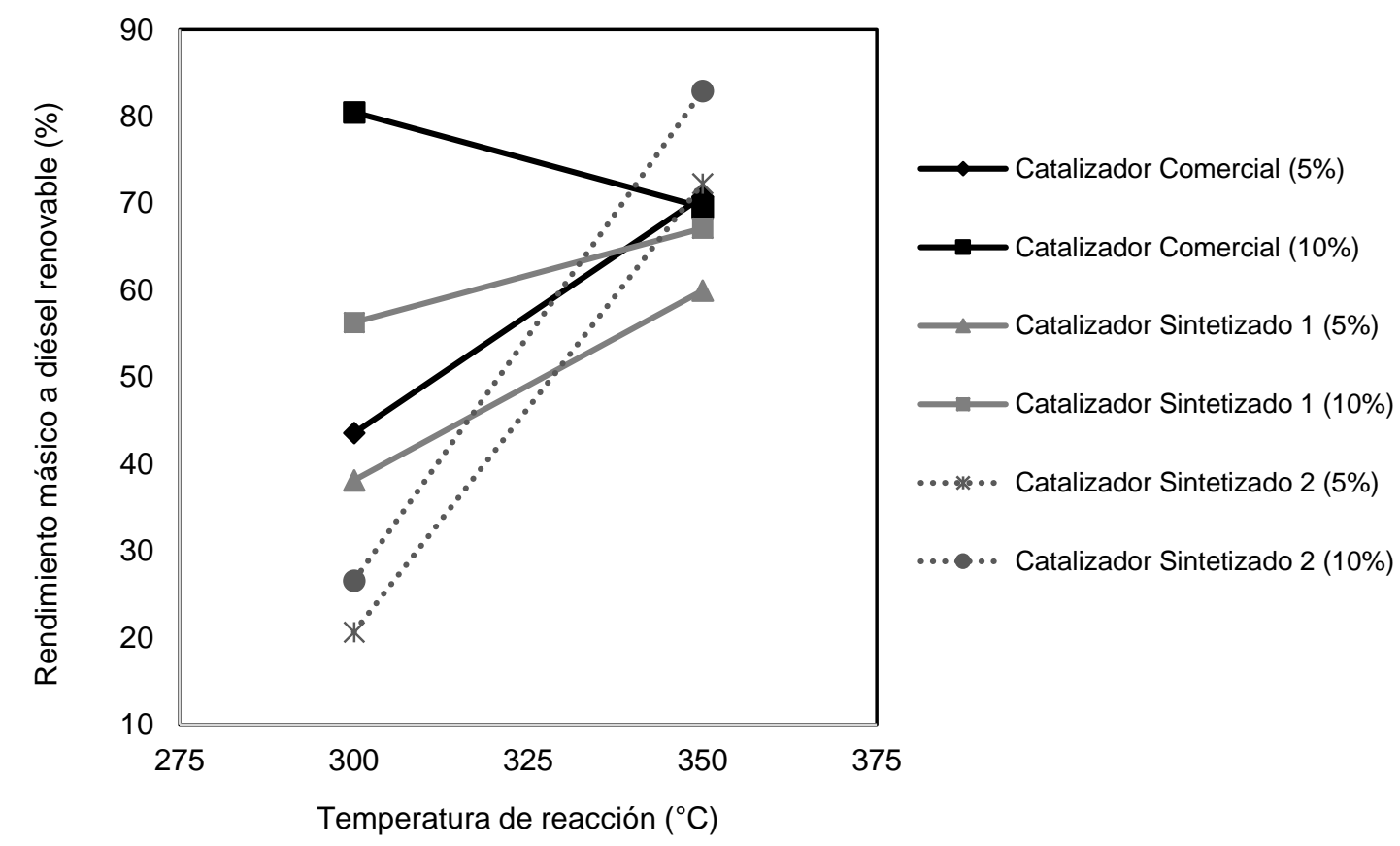

Fig. 3: Efecto de la temperatura de reacción, la concentración y tipo de catalizador en el rendimiento másico a diésel renovable. 


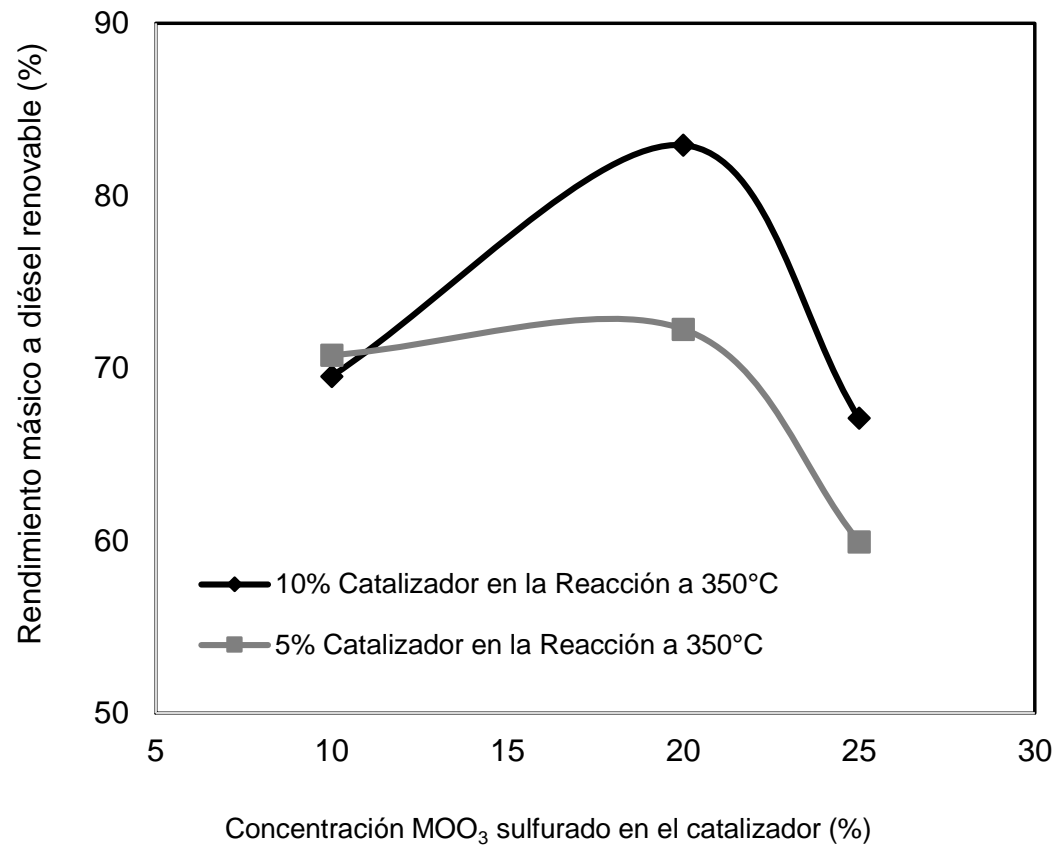

Fig. 4: Efecto de la concentración de sulfuros de molibdeno $\left(\mathrm{MoO}_{3}\right)$ en el rendimiento a diésel renovable.

A pesar de los pocos puntos experimentales, la figura 4 sugiere que en relación al contenido de la fase activa, sulfuro de molibdeno, existe un máximo a partir del cual un incremento en el contenido de esta fase es detrimental con respecto al rendimiento hacia el producto de interés. Un porcentaje inferior de esta fase (catalizador comercial) si bien permitió obtener rendimientos satisfactorios, no evidenció el mejor desempeño. Este resultado es bastante interesante puesto que este contenido de fase activa $\left(20 \% \mathrm{MoO}_{3}\right)$ pertenece al catalizador sintetizado 2, cuya área superficial es de $80 \mathrm{~m}^{2} / \mathrm{g}$, tres veces inferior a la que exhibe el catalizador comercial $\left(248 \mathrm{~m}^{2} / \mathrm{g}\right)$. La conclusión directa que se extrae es que el contenido de Mo tiene un mayor efecto en el rendimiento hacia diésel renovable que el área superficial. No obstante, un mayor contenido de esta fase, catalizador sintetizado 1 (25 \% MoO $)$, favorece la formación de productos craqueados y polímeros, a cualquier condición de reacción, tal y como se observa en las tablas 2 y 3.

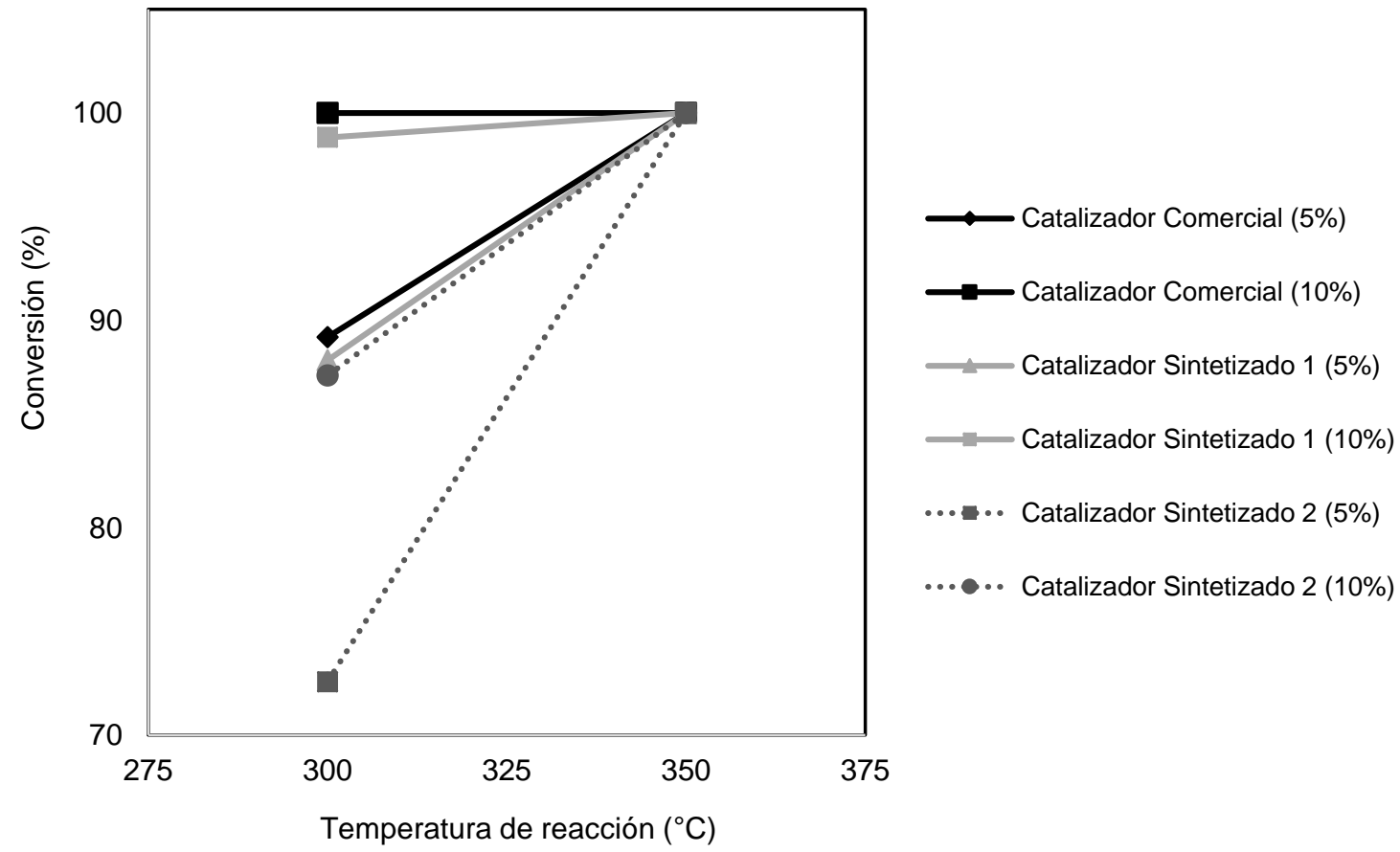

Fig. 5: Efecto de la temperatura de reacción en la conversión del aceite de higuerilla empleando diferentes catalizadores sulfurados. 
Se obtuvieron conversiones del $100 \%$ en las reacciones evaluadas a condiciones de $350^{\circ} \mathrm{C}$ y a concentraciones de catalizador del $5 \%$ y del $10 \%$ para todos los sistemas catalíticos estudiados, mientras que en los sistemas evaluados a $300^{\circ} \mathrm{C}$, el valor para esta variable fue menor en comparación con la anterior, excepto para el punto evaluado al $10 \%$ del catalizador comercial donde hubo conversión completa. Este comportamiento se presenta en la figura 5 . La conversión a $300^{\circ} \mathrm{C}$ fue del $100 \%$ para el catalizador comercial debido a su alta área superficial y contenido de fases activas, mientras que el catalizador sintetizado 1 con $25 \%$ de $\mathrm{MoO}_{3}$ mostró también una alta conversión, pero la formación de productos craqueados y polímeros disminuyeron su selectividad y rendimiento hacia diésel renovable.

Puede concluirse que existe un compromiso entre el contenido de fases activas, la forma sulfurada de las especies Ni y Mo, y la temperatura de reacción del sistema, así como la concentración de catalizador. Una alta área superficial y una temperatura de reacción de $300^{\circ} \mathrm{C}$ permitieron obtener un producto de buena calidad, pero un mayor contenido de dichas especies a una mayor temperatura mostró un efecto sinérgico que permitió obtener un producto con una alta pureza y rendimiento, incluso con un área superficial muy inferior. El contenido de $\mathrm{Ni}$ es importante para la etapa de hidrogenación y como promotor de las especies sulfuradas de Mo en la etapa de desoxigenación, incrementando la actividad catalítica (Kubička y Kaluža, 2010).

\section{CONCLUSIONES}

Se sintetizaron dos catalizadores tipo $\mathrm{NiMo} / \gamma-\mathrm{Al}_{2} \mathrm{O}_{3}$ con diferente contenido de las especies $\mathrm{Ni}$ y $\mathrm{Mo}$ (catalizador $1: 6 \% \mathrm{NiO}$ y $25 \% \mathrm{MoO}_{3}$ y catalizador $2: 4,5 \% \mathrm{NiO}$ y $20 \% \mathrm{MoO}_{3}$ ), y su actividad catalítica en la reacción de desoxigenación del aceite de higuerilla fue evaluada bajo diferentes condiciones experimentales y comparada con las propiedades catalíticas de un catalizador comercial $\left(3,0 \% \mathrm{NiO}\right.$ y $\left.10 \% \mathrm{MoO}_{3}\right)$.

La caracterización efectuada a los tres catalizadores permitió concluir que están presentes las especies activas de $\mathrm{Mo}\left(\mathrm{MoS}_{2}\right)$, y que el área superficial, el volumen de poro y el diámetro de poro tienen valores suficientes para permitir la difusión de las moléculas reaccionantes, aunque este último aspecto requiere mayor profundización. El estudio de XRF mostró que el proceso de impregnación de los metales y que la sulfuración del catalizador fue exitosa, y permitió establecer los rangos de concentración de las especies de interés. Los perfiles TPR fueron similares para los tres catalizadores, pero la intensidad de los picos fue diferente y se incrementó en función del contenido de Molibdeno. Se observaron las reducciones $\mathrm{Mo}^{6+} \rightarrow \mathrm{Mo}^{4+}, \mathrm{Mo}^{4+} \rightarrow \mathrm{Mo}^{0}$ y Ni${ }^{2+} \rightarrow \mathrm{Ni}^{0}$.

De forma general se puede concluir que se obtuvieron altas conversiones y rendimientos hacia el producto de interés, diésel renovable, a condiciones típicas de desoxigenación: $350{ }^{\circ} \mathrm{C}, 90$ bares de presión de $\mathrm{H}_{2}$ y 4 h de reacción. El mejor rendimiento (82,9\%) y selectividad hacia diésel renovable $(82,9 \%)$, así como la mayor pureza $(94,8 \%)$, se obtuvieron con el catalizador sintetizado 2 operando a $350^{\circ} \mathrm{C}, 10 \%$ de catalizador, 90 bar de presión, 350 rpm y 4 horas de reacción en sistema semi-batch. Bajo estas condiciones la conversión del aceite de higuerilla fue del $100 \%$.

La selectividad hacia heptadecano en todos los casos fue mayor que hacia octadecano, lo que indica que la mayoría de los productos de la reacción se están formando por las rutas descarboxilación $\left(\mathrm{DCO}_{2}\right)$, descarbonilación (DCO) y en menor porcentaje por hidrodesoxigenación (HDO). Los factores que tuvieron una mayor incidencia sobre la pureza y el rendimiento en la obtención de diésel renovable fueron el contenido de fases activas, especialmente la forma sulfurada de las especies de niquel y molibdeno, la temperatura de reacción del sistema y la concentración de catalizador.

\section{AGRADECIMIENTOS}

Los autores agradecen a Colciencias y al "Patrimonio Autónomo Fondo Nacional de Financiamiento para la Ciencia, la Tecnología y la Innovación, Francisco José de Caldas"; así como a la "Estrategia de sostenibilidad para grupos en consolidación 2016-2017" y al "Comité para el Desarrollo de la Investigación" de la Universidad de Antioquia.

\section{NOTACIÓN}

Diésel Renovable (DR):

Craqueados (C):

Polímeros (P):

Aceite Alimentado (AA):
Producto comprendido entre 9 y 24 átomos de carbono

Productos con menos de 15 átomos de carbono $\left(<\mathrm{C}_{15}\right)$

Productos con más de 20 átomos de Carbono $\left(>\mathrm{C}_{20}\right)$

Aceite de higuerilla cargado al reactor 


$\begin{array}{ll}\text { Producto Liquido (PL): } & \text { Producto líquido obtenido después de la reacción } \\ \text { YDR: } & \text { Rendimiento másico hacia Diésel Renovable (\%) } \\ \text { YPL: } & \text { Rendimiento másico de productos líquidos (\%) } \\ \text { YC: } & \text { Rendimiento másico hacia productos craqueados (\%) } \\ \text { YP: } & \text { Rendimiento másico hacia polímeros (\%) } \\ \text { C: } & \text { Conversión (\%) } \\ \text { IS AA: } & \text { Índice de saponificación del aceite de higuerilla cargado al reactor } \\ \text { IA AA: } & \text { Índice de acidez del aceite de higuerilla cargado al reactor } \\ \text { g AA: } & \text { Gramos de aceite de higuerilla cargado al reactor } \\ \text { IS PL: } & \text { Índice de saponificación del producto líquido obtenido después de la reacción } \\ \text { IA PL: } & \text { Índice de acidez del producto líquido obtenido después de la reacción } \\ \text { g PL: } & \text { Gramos de producto líquido obtenido después de la reacción } \\ \text { SDR: } & \text { Selectividad hacia Diésel Renovable (\%) }\end{array}$

\section{REFERENCIAS}

Domínguez-Crespo, M.A., Arce-Estrada, E.M., Torres-Huerta, A.M., Díaz-García, L. y Cortez de la Paz, M.T., Support effects on hydrotreating activity of NiMo catalysts, doi:10.1016/j.matchar.2006.08.013, Materials Characterization, 58, 864-873 (2007)

Donnis, B., Egeberg, R.G., Blom P. y Knudsen, K.G., Hydroprocessing of Bio-Oils and Oxygenates to Hydrocarbons. Understanding the Reaction Routes, doi: 10.1007/s11244-008-9159-z, Topics in Catalysis, 52(3), 229-240 (2009)

Duminda, A.G. y Sandun, D.F., Methods and Applications of Deoxygenation for the Conversion of Biomass to Petrochemical Products, doi: 10.5772/53983, Biomass Now - Cultivation and Utilization, InTech, Chapter 11, 273-298 (2013)

El-Ashry, M.T., National Policies to Promote Renewable Energy, doi: 10.1162/DAED_a_00150, Daedalus, 141(2), 105-110 (2012)

Gong, S., Shinozaki, A., Shi, M., y Qian, E.W., Hydrotreating of Jatropha Oil over Alumina Based Catalysts, doi: 10.1021/ef300047a, Energy \& Fuels, 26(4), 2394-2399 (2012)

Huber, G.W., O'Connor, P. y Corma, A., Processing Biomass in Conventional Oil Refineries: Production of High Quality Diesel by Hydrotreating Vegetable Oils in Heavy Vacuum OIL mixtures, doi: 10.1016/j.apcata.2007.07.002, Applied Catalysis A :General, 329, 120-129 (2007)

Kiatkittipong, W., Phimsen, S., Kiatkittipong, K., Wongsakulphasatch, S., Laosiripojana, N. y Assabumrungrat, S., Diesel-like Hydrocarbon Production from Hydroprocessing of Relevant Refining Palm Oil, doi: 10.1016/j.fuproc.2013.04.018, Fuel Processing Technology, 116, 16-26 (2013)

Kubička, D. y Horáček J., Deactivation of HDS Catalysts in Deoxygenation of Vegetable Oils, doi:10.1016/j.apcata.2010.10.034, Applied Catalysis A: General, 394(1-2), 9-17 (2011)

Kubička, D. y Kaluža L., Deoxygenation of Vegetable Oils over Sulfided Ni, Mo and Ni-Mo Catalysts, doi:10.1016/j.apcata.2009.10.034, Applied Catalysis A: General, 372(2), 199-208 (2010)

Lai, W., Pang, L., Zheng, J., Li, J., Wu, Z., Yi, X., Fang, W.y Jia, L., Efficient one pot synthesis of mesoporous $\mathrm{NiMo}_{-} \mathrm{Al}_{2} \mathrm{O}_{3}$ catalysts for dibenzothiophene hydrodesulfurization, doi:10.1016/j.fuproc.2013.01.006, Fuel Processing Technology, 110, 8-16 (2013)

Liu, J., Fan, K., Tian, W., Liu, C. y Rong, L., Hydroprocessing of Jatropha Oil over Ni-MoCe/Al $\mathrm{O}_{3}$ Catalyst, doi: 10.1016/j.ijhydene.2012.09.020, International Journal of Hydrogen Energy, 37(23), 17731-17737 (2012)

Liu, S., Zhu, Q., Guan, Q., He, L. y Li, W., Bio-aviation Fuel Production from Hydroprocessing Castor Oil Promoted by the Nickel-based Bifunctional Catalysts, doi: 10.1016/j.biortech.2015.02.056, Bioresource Technology, 183, 93-100 (2015)

Lukić, I., Krstić, J., Jovanović, D. y Skala, D., Alumina/silica supported $\mathrm{K}_{2} \mathrm{CO}_{3}$ as a catalyst for biodiesel synthesis from sunflower oil, doi:10.1016/j.biortech.2009.04.057, Bioresource Technology, 100, 4690-4696 (2009) 
Marker, T.L., Opportunities for Biorenewables in Oil Refineries, (en la web: http://www.osti.gov/scitech/biblio/861458, acceso 15 de Junio de 2016), UOP, USA (2005)

Meller, E., Green, U., Aizenshtat, Z. y Sasson, Y., Catalytic Deoxygenation of Castor Oil over Pd/C for the Production of cost Effective Biofuel, doi: 10.1016/j.fuel.2014.04.094, Fuel, 133(9), 89-95 (2014)

Moncada, J., Cardona, C.A. y Rincón, L.E., Design and analysis of a second and third generation biorefinery: The case of castorbean and microalgae, doi: 10.1016/j.biortech.2015.09.077, Bioresource Technology, 198, 836-846 (2015)

No, S.Y., Application of Hydrotreated Vegetable Oil from Triglyceride Based Biomass to Cl Engines - A Review, doi: 10.1016/j.fuel.2013.07.00, Fuel, 115, 88-96 (2014)

NTC 218, Grasas y Aceites Vegetales y Animales, Determinación del Índice de Acidez y de la Acidez, 1-14, Bogotá, D.C., Colombia (2011)

NTC 335, Grasas y Aceites Vegetales y Animales, Determinación del Índice de Saponificación, 1-7, Bogotá, D.C., Colombia (1998)

Ogunkoya, D., Roberts, W.L., Fang, T., Thapaliya, N., Investigation of the effects of renewable diesel fuels on engine performance, combustion, and emissions, doi: 10.1016/j.fuel.2014.09.061, Fuel, 140, 541-554 (2015)

Olusola, J.O., Adediran, M.M., Oluseyi, A.K. y Ajao, U.L., Processing of Triglycerides to Diesel Range Hydrocarbon Fuels: Easily Practicable Small Scale Approach, doi: 10.1260/0958-305X.20/21.8/1.1325, Energy \& Environment, 20/21(8/1), 1325 -1341 (2010)

Priecel, P., Kubička, D., Čapek, L., Bastl, Z. y Rysánek, P., The Role of Ni Species in the Deoxygenation of Rapeseed Oil over NiMo-Alumina Catalysts, doi:10.1016/j.apcata.2011.02.022, Applied Catalysis A: General, 397(1-2), 127-137 (2011a)

Priecel, P., Čapek, L., Kubička, D., Homolab, F., Rysánek, P. y Pouzar, M., The Role of Alumina Support in the Deoxygenation of Rapeseed Oil over Ni-Mo-Alumina Catalysts, doi:10.1016/j.cattod.2010.11.022, Catalysis Today, 176(1), 409-412 (2011b)

Rabaev, M., Landau, M.V., Vidruk-Nehemya, R., Koukouliev, V., Zarchin, R. y Herskowitz, M., Conversion of Vegetable Oils on Pt/AI2O3/SAPO-11 to Diesel and Jet Fuels Containing Aromatics, doi: 10.1016/j.fuel.2015.08.063, Fuel, 161, 287-294 (2015)

Romero, M., Pizzi, A., Toscano, G., Casazza, A.A., Busca, G., Bosio, B. y Arato, E., Preliminary Experimental Study on Biofuel Production by Deoxygenation of Jatropha Oil, doi: 10.1016/j.fuproc.2015.04.002, Fuel Processing Technology, 137, 31-37 (2015)

Sebos, I., Matsoukas, A., Apostolopoulos, V. y Papayannakos, N., Catalytic Hydroprocessing of Cottonseed Oil in Petroleum Diesel Mixtures for Production of Renewable Diesel, doi:10.1016/j.fuel.2008.07.032, Fuel, 88(1), 145-149 (2009)

Šimáček, P., Kubička, D., Kubičkova, I., Homola, F., Pospíšil, M. y Chudoba, J., Premium Quality Renewable Diesel Fuel by Hydroprocessing of Sunflower Oil, doi:10.1016/j.fuel.2011.03.013, Fuel, 90(7), 2473-2479 (2011)

Srifa, A., Faungnawakij,K., Itthibenchapong, V., Viriya-empikul, N., Charinpanitkul, T. y Assabumrungrat, S., Production of bio-hydrogenated diesel by catalytic hydrotreating of palm oil over Ni-MoS $\mathrm{N}_{2} \mathrm{~V}-\mathrm{Al}_{2} \mathrm{O}_{3} \mathrm{Catalyst}_{\text {, }}$ doi: 10.1016/j.biortech.2014.01.100, Bioresource Technology, 158, 81-90 (2014)

Veriansyah, B., Han, J.Y., Kim, S.K., Hong, S-A., Kim, Y.J., Lim, J.S., Shu,Y-W., Oh, S-G. y Kim, J., Production of Renewable Diesel by Hydroprocessing of Soybean oil: Effect of catalysts, doi:10.1016/j.fuel.2011.10.057, Fuel, 94, 578-585 (2012)

Wang, J., Chao, D., Liu, J., Li, L., Lai, L., Lin, J. y Shen, Z., Ni3 $S_{2} @ M o S_{2}$ core/shell nanorod arrays on Ni foam for high-performance electrochemical energy storage, doi:10.1016/j.nanoen.2014.04.019, Nano Energy (7), 151-160 (2014) 